\title{
How To Weaken Zika Virus?
}

\author{
Zheng Liu \\ School of North China Electric Power University, Baoding 071003, China \\ hbxt3281510@163.com
}

Keywords: SEIR, differential equations, delay differential equations, spreading trend.

\begin{abstract}
This paper mainly focuses on providing a plan for using Wolbachia to influence the mosquito population with Zika Virus. In our SEIR Model we first obtain and solve a series of differential equations to predict the tread of Zika spreading, based on this model, we bulid a Wolbachia Spread In Mosquitoes Model to find how Wolbachia invades the population of mosquito. By building a series of delay differential equations, we successfully predict the Zika virus spreading trend after introducing modified mosquitoes. The results shows that at least 450000 modified mosquitoes are needed to meet the constraints.
\end{abstract}

\section{Introduction}

Zika virus is an arbovirus transmitted by Aedes aegypti mosquito, Its incubation period (from exposure to symptoms) is not clear, may be a few days ${ }^{[1]}$. Only about $20 \%$ of those infected with the virus will show mild symptoms.However, pregnant women are infected, often lead to severe infant defects(as shown in Figure 1).

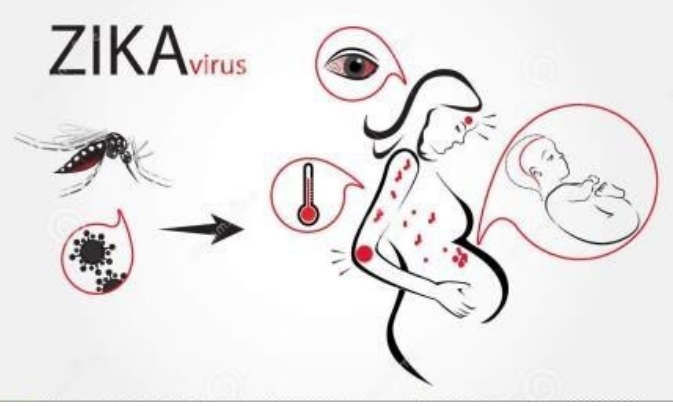

Fig. 1: How Zika virus spread by mosquitoes

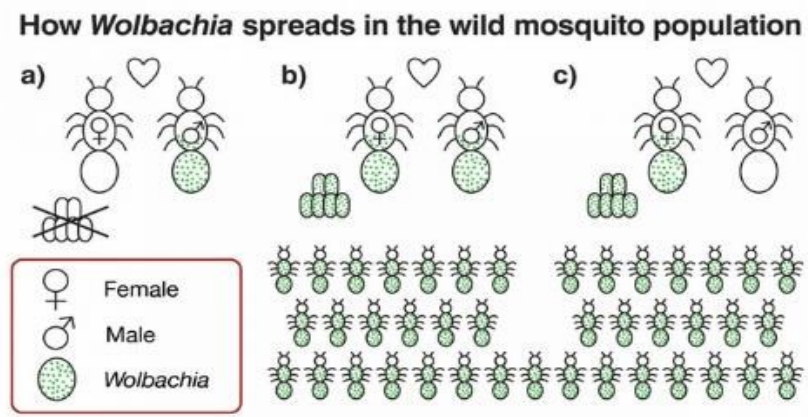

Fig. 2: How Wolbachia spread in mosquitoes

At present, there is no vaccine and prevention technology for the Zika virus, So in addition to man-made measures to reduce mosquito bites, mosquitoes carrying the Wolbachia bacterium, which growing in the laboratory ${ }^{[2]}$, release into the environment. It can reduce the population of mosquitoes and mosquitoes' capacity to transmit Zika to humans(as shown in Figure 2).

\section{Model Theory}

\subsection{The SEIR Model}

In this model, human population of Puerto Rico is divided into six classes: susceptible $\left(S_{h}(t)\right)$, exposed $\left(E_{h}(t)\right)$, symptomatically infected $\left(I_{h 1}(t)\right)$, convalescent $\left(I_{h 2}(t)\right)$ and recovered $\left(R_{h}(t)\right)^{[3]}$ at time $t>0$, and the mosquito population is divided into three parts: susceptible $\left(S_{v}(t)\right)$, exposed $\left(E_{v}(t)\right)$, and infectious $\left(I_{v}(t)\right)$, respectively. We mark $N_{h}$ as the total number of humans, $N_{v}$ as the total mosquito population, so:

$$
N_{h}=S_{h}+E_{h}+I_{h}+R_{h}
$$

If we assume Nv as the total mosquito population,there is:

$$
N_{v}=S_{v}+E_{v}+I_{v}
$$


Both $N_{h}$ and $N_{v}$ are assumed to be constant. We use the SEI type of structure for mosquitoes and SEIR type of structure for humans. Based on the assumptions, the ZIKV transmission dynamics between humans and mosquitoes are governed by the following model equations:

$$
\begin{aligned}
& \frac{d S_{h}}{d t}=-a b \frac{I_{v}}{N_{h}} S_{h}-\beta \frac{\kappa E_{h}+I_{h}}{N_{h}} S_{h} \\
& \frac{d E_{h}}{d t}=\theta\left(a b \frac{I_{v}}{N_{h}} S_{h}+\beta \frac{\kappa E_{h}+I_{h}}{N_{h}} S_{h}\right)-V_{h} E_{h} \\
& \frac{d I_{h}}{d t}=V_{h} E_{h}-\gamma_{h 1} I_{h} \\
& \frac{d R_{h}}{d t}=\gamma_{h 2} I_{h} \\
& \frac{d S_{v}}{d t}=\mu_{v} N_{v}-a c \frac{\eta E_{h}+I_{h 1}}{N_{h}} S_{v}-\mu_{v} S_{v} \\
& \frac{d E_{v}}{d t}=a c \frac{\eta E_{h}+I_{h}}{N_{h}} S_{v}-\left(V_{v}+\mu_{v}\right) E_{v} \\
& \frac{d I_{v}}{d t}=v_{v} E_{v}-\mu_{v} I_{v}
\end{aligned}
$$

Where:

$a$ is the mosquito biting rate.

$b$ is the transmission probability from an mosquito to a susceptible human per bite.

$\beta$ is the transmission rate from symptomatically infected humans to susceptible humans. $\kappa$ is the relative human-to-human transmissibility of exposed humans to symptomatic. $\theta$ is the proportion of symptomatic infections.

$\eta$ is the relative human-to-mosquito transmission probability of exposed to infected $1 / \mu_{\mathrm{v}}$ is the mosquito lifespan. ( Days )

\subsection{Results of the SEIR Model}

To solve the further questions, we first predict how the numbers of the infected public (as shown in Figure 3) and pregnant women (as shown in Figure 4) value with time going.

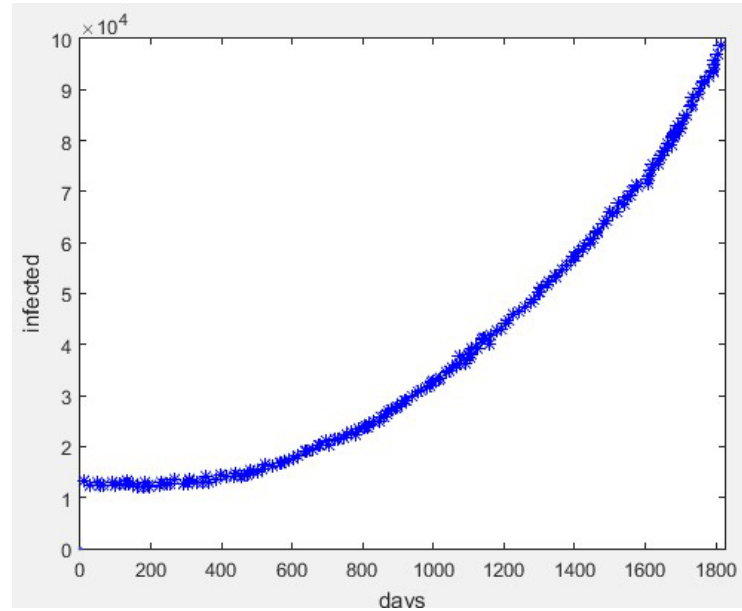

Fig. 3: Trend of the infected's number

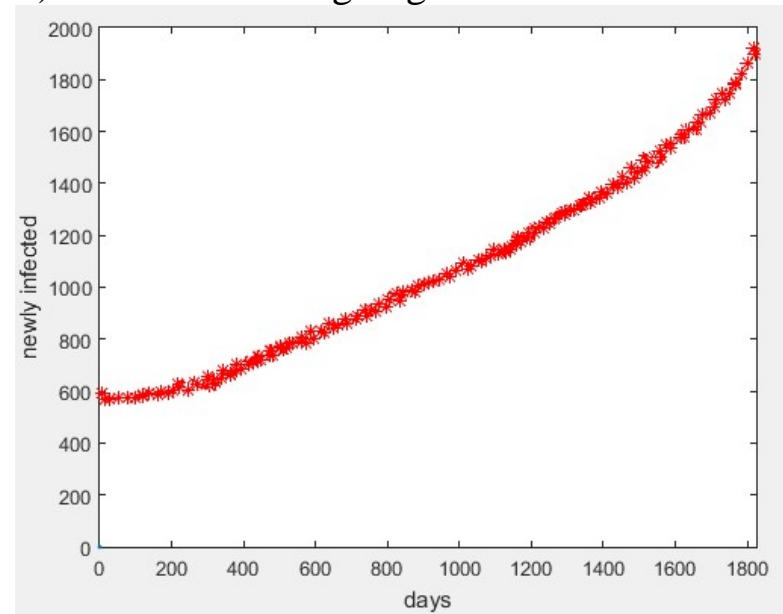

Fig. 4: Trend of infected pregnant women's number

From the figure above, we can see that the number of the infected rise fast under no control. Therefore, it's necessary to introduce some "modified mosquitoes" to improve this situation.

\subsection{Results of the SEIR Model}

In our model, we consider the dynamics of reproductive adult mosquitoes. Let $R_{F}(t)$ denote the number of released females at time $\mathrm{t}$, and $R_{M}(t)$ the number of released males, both infected. We 
assume that the decay rate for either $R_{F}(t)$ or $R_{M}(t)$ increases with its own size and the total population size $N_{v}(t)$ due to strong competition between adults ${ }^{[4]}$. Let $\delta_{I}$ be the decay rate constant. Then

$$
\begin{aligned}
& \frac{d R_{F}(t)}{d t}=-\delta_{I} R_{F}(t) N_{v}(t) \\
& \frac{d R_{M}(t)}{d t}=-\delta_{I} R_{M}(t) N_{v}(t)
\end{aligned}
$$

Let $R_{F}(t)$ and $R_{M}(t)$ denote the numbers of uninfected reproductive females and males, $R_{F}(t)$ and $R_{M}(t)$ denote the numbers of infected reproductive females and males other than those from releasing, respectively. Then the total number $N_{v}(t)$ has the decomposition

$$
N_{v}(t)=I_{F}(t)+I_{M}(t)+U_{F}(t)+U_{M}(t)+R_{F}(t)+R_{M}(t)
$$

The same, mosquitoes that are susceptible become mild after being infected by the Wolbachia.

$$
\begin{gathered}
\frac{S_{V}(t)}{d t}=\left(\mu_{V} N_{V}-a c \frac{\eta E_{h}+I_{h}}{N_{h}} S_{V}-\mu_{V} S_{V}\right) \frac{U_{F}(t)+U_{M}(t)+R_{F}(t)+R_{M}(t)}{N_{V}(t)} \\
\frac{E_{V}(t)}{d t}=\left(a c \frac{\eta E_{h}+I_{h}}{N_{h}} S_{V}-(v) \mu_{V} S_{V}\right) \frac{U_{F}(t)+U_{M}(t)+R_{F}(t)+R_{M}(t)}{N_{V}(t)}
\end{gathered}
$$

To meet the constraints, we analysis how the ratio of Wolbachia-infected mosquitoes change while the take $\tau$ different values.

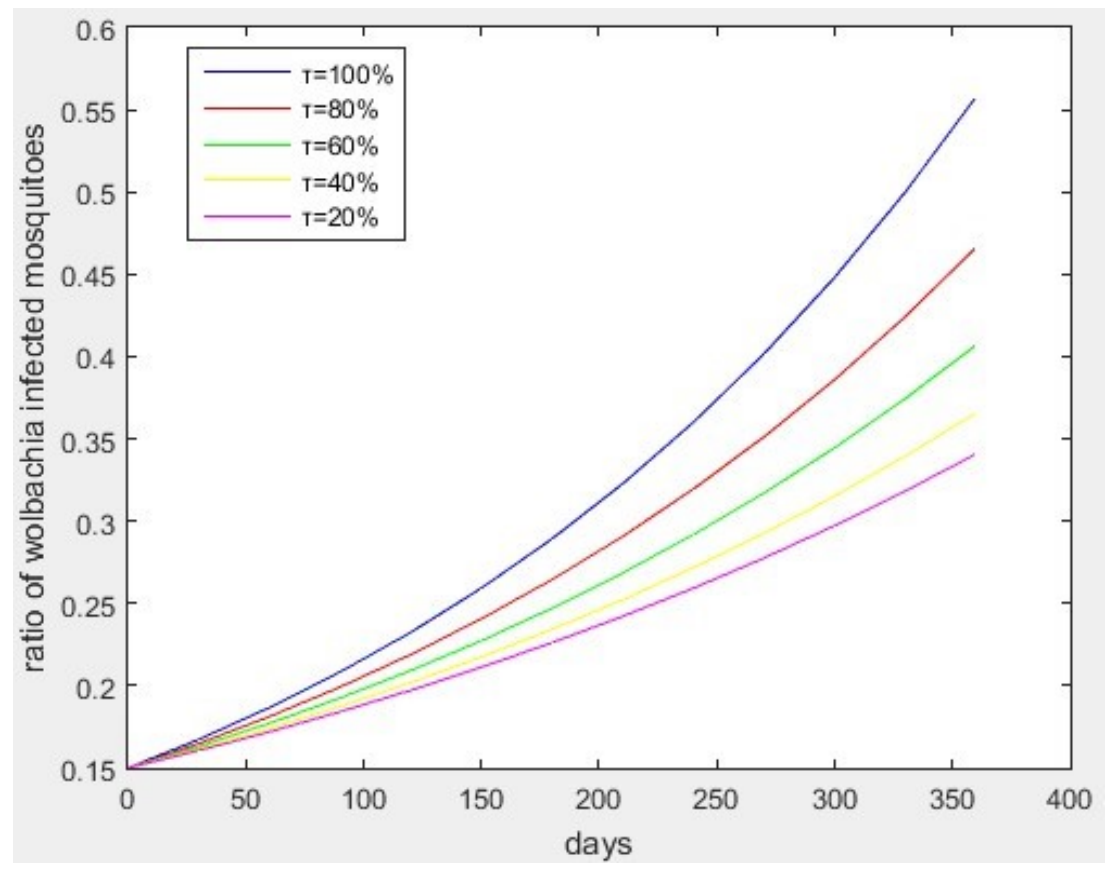

Fig. 5: The ratio of $\mathrm{mW} / \mathrm{fW}$ under different value

In the figure 5 above, the blue, orange and green curve represents the values of $\tau$, the ratio of $\mathrm{mW} / \mathrm{fW}$, they are respectively $20 \%, 40 \%, 60 \%, 80 \%, 100 \%$. In the picture, we find that the ratio of Wolbachia-infected mosquitoes rise fastest when the $\tau$ reach 1 . Therefore, we can combine the parameters blow and the differential equations above to predict the trend that Zika spread in the Puerto Rico ${ }^{[6]}$ (As shown in picture 6) 

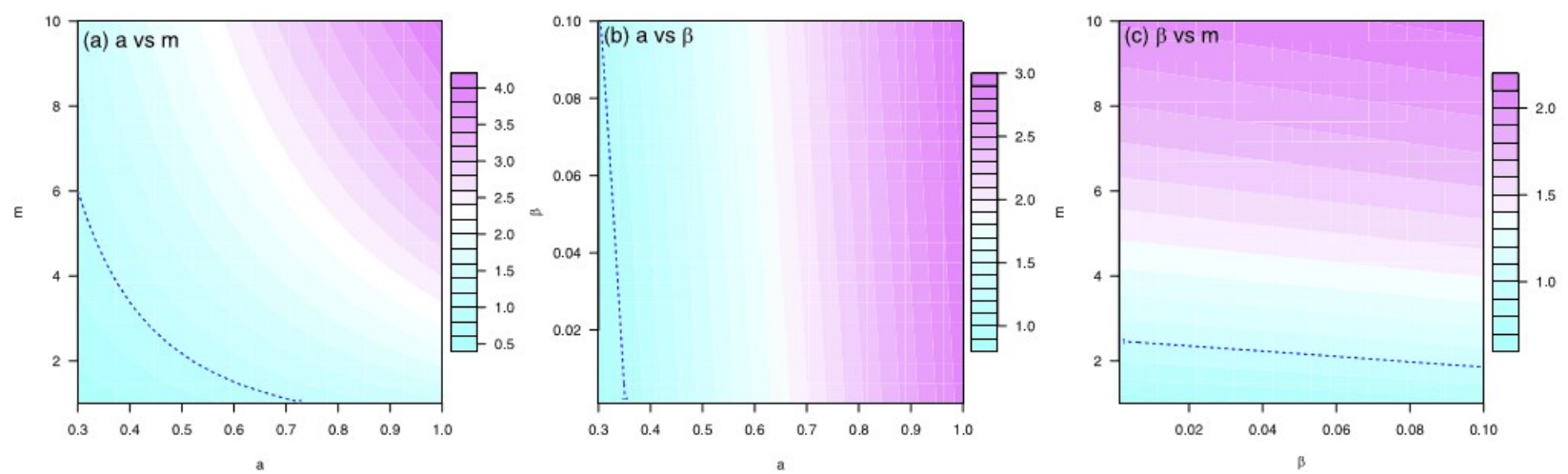

Fig 6: The contour plot of the basic reproduction number in the three controllable parameters

\section{Conclusion}

According to the message, as of right now, the number of newly infected Puerto Ricans is estimated to be 600 per day, including 22 pregnant women, then, we can reach the total number of Zika infected public after 5 years.

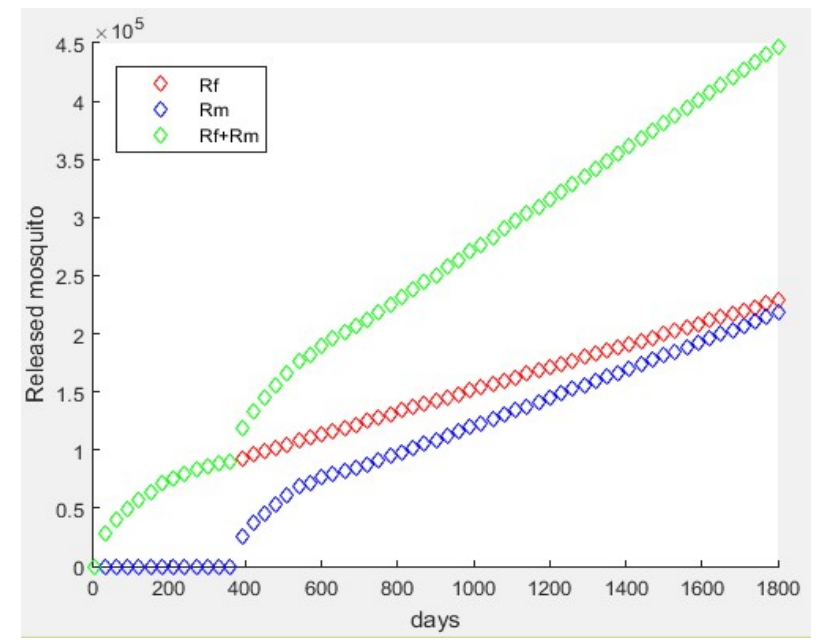

Fig.7: The number of released Wolbachia -Infected female (male) mosquito at time $\mathrm{t}$

\section{References}

[1] Duffy, M. R. et al. Zika virus outbreak on Yap Island, Federated States of Micronesia. New Engl. J. Med. 360(24), 2536-2543 (2009).

[2] Mlakar, J. et al. Zika virus associated with microcephaly. New Engl. J. Med. 374(10), 951-958 (2016).

[3] Cauchemez, S. et al. Association between Zika virus and microcephaly in French Polynesia, 2013-15: a retrospective study, Lancet Published online March 15, 2016. http://dx.doi.org/10.1016/S0140-6736(16)00651-6.

[4] Cao-Lormeau, V.-M. et al. Guillain-Barré Syndrome outbreak associated with Zika virus infection in French Polynesia: a casecontrol study. Lancet 387(10027), 1531-1539 (2016).

[5] Dick, G. W., Kitchen, S. F. \& Haddow, A. J. Zika virus. I. Isolations and serological specificity. Trans. R. Soc. Trop. Med. Hyg. 46(5), 509-520 (1952).

[6] Macnamara, F. N. Zika virus: a report on three cases of human infection during an epidemic of jaundice in Nigeria. Trans. R. Soc. Trop. Med. Hyg. 48(2), 139-145 (1954). 